\title{
Caracterização da madeira de quatro espécies florestais
}

\author{
Characterization of wood of four forest species
}

\author{
Javan Pereira Motta ${ }^{\mathrm{I}}$ José Tarcísio da Silva Oliveira ${ }^{\mathrm{II}}$ \\ Rafael Leite Braz ${ }^{\text {II }}$ Ana Paula Coelho Duarte ${ }^{\text {II }}$ Rejane Costa Alves ${ }^{\text {IV }}$
}

\section{RESUMO}

\begin{abstract}
O conhecimento das relações existentes entre a estrutura anatômica da madeira com suas propriedades físicas ou mecânicas é importante para seu uso adequado. Este trabalho teve comoobjetivo caracterizar a anatomia microscópica e propriedades fisicas da madeira de quatro diferentes espécies florestais. A madeira estudada foi proveniente das seguintes espécies: Cedrela fissilis (Cedro), Híbrido clonal Eucalyptus urophylla $\times$ Eucalyptus grandis (Eucalipto urograndis), Hovenia dulcis (Uva do Japão) e Gallesia integrifolia (Pau d'alho). Para a determinação da retratibilidade, utilizou-se a norma MB26/40 da ABNT (1940); as dimensões lineares foram obtidas com palmer, precisão de 0,001 mm. O estudo da anatomia da madeira seguiu as recomendações da norma de procedimento em estudos de anatomia de madeira, da COPANT (1974) e também da IAWA (1989). Os caracteres anatômicos avaliados foram: diâmetro tangencial ( $\mu \mathrm{m})$ e frequência de vasos $\left(n^{\mathrm{o}} \mathrm{mm}^{-2}\right)$; comprimento de fibra $(\mu \mathrm{m})$, diâmetro de lume ( $\mu \mathrm{m})$ e espessura de parede de fibra ( $\mu \mathrm{m})$; altura $(\mu \mathrm{m})$, largura $(\mu \mathrm{m})$ e frequência de raios $\left(n-\mathrm{mm}^{-1}\right)$. Os valores do coeficiente anisotrópico foram de 1,16; 1,77; 1,81 e 1,84 para a madeira de $\boldsymbol{C}$. fissilis, H. dulcis, Híbrido clonal e G. integrifolia, respectivamente, demonstrando que a madeira de $\boldsymbol{C}$. fissilis possui elevada estabilidade dimensional e as demais madeiras, valores aceitáveis para esse coeficiente.
\end{abstract}

Palavras-chave: propriedades da madeira, estabilidade dimensional, qualidade.

\section{ABSTRACT}

This study aimed to characterize the microscopic anatomy and physical properties of wood from four different forest species. The wood has been studied from the following species: Cedrela fissilis, Crossbreed clone Eucalyptus urophylla

\begin{abstract}
$\times$ Eucalyptus grandis, Hovenia dulcis and Gallesia integrifolia. To determine the shrinkage it was used the standard of MB26/40 of ABNT (1940), the linear dimensions were obtained with palmer, accuracy of $0.001 \mathrm{~mm}$. The study of wood anatomy followed the recommendations of standard procedure in studies of anatomy of wood, COPANT (1974) and also the IAWA (1989). The anatomical characters evaluated were: tangential diameter ( $\mu \mathrm{m})$ and frequency of vessels $\left(n^{\circ} m^{-2}\right)$, fiber length $(\mu \mathrm{m})$, diameter of lumen $(\mu \mathrm{m})$ and wall thickness of fiber $(\mu \mathrm{m})$, height $(\mu \mathrm{m})$, width $(\mu \mathrm{m})$ and frequency of rays $\left(n^{\underline{a}} \mathrm{~mm}^{-1}\right)$. The anisotropic factor values were of 1.17, 1.83, 1.89 and 1.90 for C. fissilis, H. dulcis, Crossbreed clone and $\boldsymbol{G}$. integrifolia, respectively, showing that the $\boldsymbol{C}$. fissilis wood has high stability dimensional and wood other acceptable values for this coefficient.
\end{abstract}

Key words: wood properties, dimensional stability, quality.

\section{INTRODUÇÃO}

A avaliação das propriedades da madeira é determinante para sua correta utilização, seleção de material e qualidade do produto final. O potencial tecnológico de uma espécie florestal é verificado por meio de uma série de estudos que envolvem as caracterizações anatômica, física, mecânica e química. Além disso, identificar as diferentes madeiras das espécies florestais auxilia o comércio e a indústria madeireira a evitar fraudes (COSTA, 2001).

A qualidade dos produtos provenientes da madeira vai depender do conjunto de características

\footnotetext{
'Departamento de Tecnologia da Madeira, Universidade do Estado do Pará (UEPA), Campus VIII, Avenida Hiléia, s/n, Agrópoles do INCRA, 68503-120, Amapá, Marabá, PA, Brasil. E-mail: jpereiramotta@yahoo.com.br. Autor para correspondência.

ID Departamento de Ciências Florestais e da Madeira (DCFM), Universidade Federal do Espírito Santo (UFES), Jerônimo Monteiro, ES, Brasil. IIIDepartamento de Engenharia Florestal, Universidade Federal do Paraná (UFPR), Curitiba, PR, Brasil.

${ }^{\mathrm{IV}}$ Engenheira Industrial Madeireira, Departamento de Ciências Vegetais, Universidade Federal Rural do Semi-Árido (UFERSA), Mossoró, RN, Brasil.
} 
anatômicas, químicas, físicas e mecânicas. Estes parâmetros, ao serem avaliados, são indicativos que contribuem no emprego de determinada espécie de madeira para um fim específico (FREDERICO, 2009). A composição química e a organização dos elementos celulares da madeira determinam suas propriedades e aptidões para o uso comercial (CHIMELO, 2007).

É importante destacar que a influência da anatomia da madeira está relacionada principalmente com sua estrutura, que diz respeito às diferenças nas dimensões dos elementos celulares, bem como as disposições, dimensões e frequência das cavidades celulares, que se relacionam com a porosidade e permeabilidade da madeira (ALBUQUERQUE et al., 2005). Segundo TOMAZELLO FILHO (2002), o conhecimento das células e tecidos que compõem o lenho arbóreo é básico para a caracterização da estrutura anatômica das madeiras. As dimensões e disposição dos elementos anatômicos também contribuem para o estudo das madeiras, estabelecendo informações relacionadas aos parâmetros físicos e mecânicos.

BREMER (2009) alerta sobre a importância do conhecimento das propriedades físicas da madeira utilizada estruturalmente, pois estas propriedades podem influenciar significativamente em seu desempenho e resistência. Conhecer o comportamento tecnológico da madeira das diferentes espécies florestais, nativas ou exóticas, é fundamental para aumentar as alternativas de uso delas.

LATORRACA \& ALBUQUERQUE (2000) e CRUZ et al. (2003) mencionaram que a densidade é a característica mais utilizada em pesquisas relacionadas à qualidade da madeira, sendo esta a mais importante e a que melhor se relaciona com as demais propriedades. LOBÃO et al. (2004) afirmaram que a resistência mecânica da madeira é influenciada diretamente pela densidade do material.

As variações dimensionais provocadas pela contração e pelo inchamento da madeira constituem, conjuntamente com a anisotropia, características indesejáveis da madeira, limitando o seu uso para diversas finalidades ou, ainda, exigindo técnicas específicas de utilização (DURLO \& MARCHIORI, 1992; SILVA \& OLIVEIRA, 2003). Nesse contexto, verifica-se a necessidade em disponibilizar informações que auxiliam na escolha e adequação de uso racional da madeira.

O presente trabalho teve como objetivo caracterizar a estrutura anatômica e as propriedades físicas da madeira de espécies florestais comercializadas no Estado do Espírito Santo. As mesmas foram selecionadas em função da ausência de informações técnicas confiáveis, por parte dos comerciantes locais, a respeito das principais características tecnológicas da madeira comercializada.

\section{MATERIAL E MÉTODOS}

Descrição do material

Para realização deste estudo, foram utilizadas madeiras das espécies florestais Cedrela fissilis, do híbrido clonal Eucalyptus urophylla $\times$ Eucalyptus grandis, Hovenia dulcis e Gallesia integrifolia, procedentes do município Jerônimo Monteiro, Estado do Espírito Santo. As madeiras foram adquiridas na forma de tábuas, com espessura nominal de $3,0 \mathrm{~cm}$ de comprimento e largura variável. Informações sobre a idade e localização das tábuas no tronco são desconhecidas. No entanto, realizou-se a amostragem para as análises no cerne periférico.

\section{Caracterização anatômica}

A caracterização anatômica foi realizada por meio da microscopia. Foram seguidas as recomendações da Comissão Panamericana de Normas Técnicas (COPANT, 1974) e da lista de características microscópicas para a identificação da madeira da Associação Internacional de Anatomistas de Madeira (IAWA, 1989).

Foram feitas fotomicrografias dos planos transversal, tangencial e radial da madeira nas lâminas temporárias, por meio de uma câmera fotográfica digital, acoplada a um microscópio óptico comum. Com auxílio de um sistema de análises de imagem provido do software, foi possível mensurar os elementos anatômicos. Para a frequência vascular ( $\mathrm{n}^{\circ}$ de células), diâmetro tangencial de poros $(\mu \mathrm{m})$, altura, largura e frequência dos raios $(\mu \mathrm{m})$, utilizaramse 200 repetições. Relativo ao comprimento, largura, diâmetro do lume e espessura de parede $(\mu \mathrm{m})$ foram utilizadas 100 repetições.

Para a mensuração das fibras, realizou-se a dissociação dos elementos anatômicos, utilizando o método proposto por NICHOLLS \& DADSWELL, descrito por RAMALHO (1987).

\section{Caracterização física}

A caracterização física foi mediante a determinação da densidade básica e da retratibilidade da madeira, por meio das contrações lineares e volumétricas. As determinações seguiram recomendações do método brasileiro MB26 da Associação Brasileira de Normas Técnicas - ABNT (1940).

A densidade básica foi determinada utilizando-se 20 repetições e os corpos de prova nas 
dimensões de $2,0 \times 2,0 \times 3,0 \mathrm{~cm}$, com a última medida no sentido das fibras. Os volumes das amostras saturadas e anidras foram obtidos pelo método de deslocamento de massa ou balança hidrostática, com substituição da água pelo mercúrio $(\mathrm{Hg})$, verificando em determinados períodos de tempo sua temperatura para efetuar correções na sua massa específica. Em seguida, a massa das amostras foi determinada em uma balança de precisão de $0,01 \mathrm{~g}$.

A contração volumétrica e as contrações lineares foram determinadas em corpos de prova orientados nos sentidos axial, radial e tangencial, com dimensões nominais de $2,0 \times 2,0 \times 3,0 \mathrm{~cm}$, sendo a última medida na direção das fibras, com um total de 20 repetições. As medidas nos sentidos radial e tangencial foram obtidas por meio de micrômetro digital, com precisão de $0,001 \mathrm{~mm}$. Em seguida, determinou-se o coeficiente de anisotropia, por meio da relação das contrações tangencial e radial.

Todos os resultados obtidos foram tabulados e interpretados, sendo encontrados os valores mínimos, máximos, a média e o coeficiente de variação. Para avaliar o grau de significância e suas variações, realizou-se uma análise de variância e, quando verificadas diferenças entre eles, procedeuse ao teste de média de Tukey a 5\% de significância.

\section{RESULTADOS E DISCUSSÃO}

\section{Caracterização anatômica microscópica}

Na tabela 1, estão apresentados os valores médios da mensuração dos caracteres anatômicos do lenho das madeiras estudadas.

Relativo à frequência vascular, verifica-se que a madeira de Cedrela fissilis foi a que apresentou menor valor médio, sendo classificada como madeira de muito poucos poros por $\mathrm{mm}^{2}$, diferenciando estatisticamente das demais, que são classificadas como madeiras de poucos poros. No entanto, para esta mesma característica, a madeira de Gallesia integrifolia, estatisticamente superior pelo teste de Tukey $(\mathrm{P}>0,05)$, apresentou valor máximo de 24 poros por $\mathrm{mm}^{2}$, podendo ser classificada como madeira de numerosos poros por $\mathrm{mm}^{2}$.

Para o diâmetro tangencial dos poros, verifica-se, na tabela 1 , que a madeira de $\boldsymbol{C}$. fissilis apresentou maior valor médio estatisticamente superior, com seu valor variando entre 108,48 e $345,78 \mu \mathrm{m}$, classificada como madeira de poros grandes. Já a madeira de $\boldsymbol{G}$. integrifolia foi classificada como de pequenos poros, por apresentar menor valor médio, estatisticamente inferior às demais pelo teste de

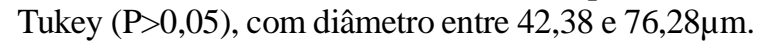

O comportamento observado acima exemplifica notadamente a relação inversa existente entre a frequência vascular e o diâmetro tangencial dos poros, como ocorre no sentido radial para as folhosas. Não foi observada diferença significativa entre as madeiras do híbrido clonal e Hovenia dulcis pelo teste de Tukey $(\mathrm{P}>0,05)$, para esta mesma característica. Estas foram classificadas como madeiras de poros médios.

Na tabela 1, com relação à altura dos raios, as madeiras de $\boldsymbol{G}$. integrifolia e $\boldsymbol{H}$. dulcis apresentaram valores médios semelhantes, superiores às demais, estatisticamente igual à madeira de $\boldsymbol{C}$. fissilis. Esta, por sua vez, foi estatisticamente igual pelo teste de Tukey $(\mathrm{P}>0,05)$ à altura dos raios da madeira do híbrido clonal, que apresentou a menor média. No entanto, todas foram classificadas como madeiras de raios pequenos.

Nota-se que, para a frequência de raios, as madeiras do híbrido clonal e $\boldsymbol{C}$. fissilis obtiveram o maior e o menor valor médios, respectivamente, estatisticamente diferentes. Portanto, a madeira do híbrido clonal é classificada como madeira de numerosos raios e a de $\boldsymbol{C}$. fissilis como de poucos raios por milímetro linear. Já as madeiras de G. integrifolia e $\boldsymbol{H}$. dulcis obtiveram valores médios estatisticamente iguais pelo teste de Tukey $(\mathrm{P}>0,05)$, sendo classificadas como madeiras de poucos raios.

Verifica-se, na tabela 1, para o comprimento das fibras, que as madeiras de $\boldsymbol{C}$. fissilis, híbrido clonal e Hovenia dulcis apresentaram os maiores valores médios, estatisticamente iguais pelo teste de Tukey $(\mathrm{P}>0,05)$, classificadas como madeiras de fibras curtas. No entanto, a madeira de $\boldsymbol{G}$. integrifolia, que apresentou a menor média para esta característica, foi estatisticamente igual à H. dulcis e do híbrido clonal, sendo, portanto, classificada como madeira de fibra muito curta.

Relativo à largura das fibras, nota-se, na tabela 1, que a madeira de $\boldsymbol{C}$. fissilis obteve maior valor médio, sendo estatisticamente superior em relação às demais, que apresentaram valores médios semelhantes, 17,$77 ; 18,32$ e 18,82, respectivamente, estatisticamente iguais pelo teste de Tukey $(\mathrm{P}>0,05)$. Nota-se que, para o diâmetro do lume, novamente a madeira de $\boldsymbol{C}$. fissilis apresentou maior valor médio estatisticamente diferente das demais madeiras, que não se diferenciaram estatisticamente pelo teste de Tukey.

Para a espessura de parede de fibra, observa-se, na tabela 1 , que os valores médios entre as madeiras foram semelhantes. No entanto, as madeiras do híbrido clonal e $\boldsymbol{G}$. integrifolia se diferenciaram estatisticamente pelo teste de Tukey $(\mathrm{P}>0,05)$. Verifica-se que, para a madeira de 
Tabela 1 - Caracteres anatômicos do cerne periférico.

\begin{tabular}{|c|c|c|c|c|c|}
\hline \multicolumn{2}{|c|}{ Caracteres Anatômicos } & \multirow{3}{*}{$\frac{\text { Cedrela fissilis }}{(0,00)(6,00)^{1}}$} & \multirow{3}{*}{$\frac{\text { Híbrido clonal }}{(6,00)(17,00)}$} & \multirow{3}{*}{$\frac{\text { Gallesia integrifolia }}{(7,00)(24,00)}$} & \multirow{3}{*}{$\frac{\text { Hovenia dulcis }}{(2,00)(14,00)}$} \\
\hline & & & & & \\
\hline \multirow{7}{*}{ Vasos } & \multirow{3}{*}{$\begin{array}{l}\text { Frequência vascular } \\
\left(n^{-} \mathrm{mm}^{-2}\right)\end{array}$} & & & & \\
\hline & & $2,68 \mathrm{~d}$ & $10,20 \mathrm{~b}$ & $13,90 \mathrm{a}$ & $6,85 \mathrm{c}$ \\
\hline & & $65,79 *$ & 21,66 & 31,79 & 39,75 \\
\hline & & & & & \\
\hline & \multirow{3}{*}{$\begin{array}{l}\text { Diâmetro tangencial } \\
(\mu \mathrm{m})\end{array}$} & $(108,48)(345,78)$ & $(71,19)(167,81)$ & $(42,38)(76,28)$ & $(55,94)(186,45)$ \\
\hline & & $224,08 \mathrm{a}$ & $127,29 b$ & $58,10 \mathrm{c}$ & $114,79 b$ \\
\hline & & 27,56 & 17,31 & 13,87 & 33,02 \\
\hline \multirow{9}{*}{ Raios } & \multirow{4}{*}{ Altura $(\mu \mathrm{m})$} & $(123,84)(400,03)$ & $(87,40)(484,77)$ & $(116,16)(557,66)$ & $(153,01)(682,81)$ \\
\hline & & $272,73 \mathrm{abc}$ & $253,98 \mathrm{c}$ & $318,61 \mathrm{ab}$ & $327,15 \mathrm{a}$ \\
\hline & & 21,33 & 35,53 & 38,09 & 31,57 \\
\hline & & $(18,65)(40,68)$ & $(5,93)(15,26)$ & $(12,71)(57,63)$ & $(32,21)(68,65)$ \\
\hline & \multirow[t]{2}{*}{ Largura $(\mu \mathrm{m})$} & $28,48 b$ & $9,05 \mathrm{c}$ & $32,08 b$ & $51,53 \mathrm{a}$ \\
\hline & & 17,11 & 21,88 & 33,16 & 16,52 \\
\hline & \multirow{3}{*}{ Frequência $\left(\mathrm{n}^{\mathrm{O}} \mathrm{mm}^{-1}\right)$} & $(4,00)(8,00)$ & $(11,00)(15,00)$ & $(4,00)(9,00)$ & $(5,00)(9,00)$ \\
\hline & & $5,60 \mathrm{c}$ & $12,85 \mathrm{a}$ & $6,60 \mathrm{~b}$ & $6,63 b$ \\
\hline & & 17,99 & 10,21 & 20,53 & 17,32 \\
\hline \multirow{13}{*}{ Fibras } & \multirow{3}{*}{ Comprimento $(\mu \mathrm{m})$} & $(705,15)(1473,51)$ & $(672,87)(1314,87)$ & $(594,67)(1126,89)$ & $(570,74)(1202,65)$ \\
\hline & & $1098,68 \mathrm{a}$ & $1013,72 \mathrm{ab}$ & $869,34 b$ & $967,86 a b$ \\
\hline & & 21,94 & 14,54 & 16,13 & 15,60 \\
\hline & \multirow{3}{*}{ Largura $(\mu \mathrm{m})$} & $(14,52)(31,68)$ & $(13,93)(21,60)$ & $(13,24)(24,67)$ & $(14,46)(22,39)$ \\
\hline & & $24,09 \mathrm{a}$ & $17,77 b$ & $18,32 b$ & $18,82 \mathrm{~b}$ \\
\hline & & 19,44 & 14,26 & 18,66 & 11,35 \\
\hline & & & & & \\
\hline & \multirow{3}{*}{$\begin{array}{l}\text { Diâmetro do lume } \\
(\mu \mathrm{m})\end{array}$} & $(6,38)(19,31)$ & $(3,85)(13,19)$ & $(4,68)(15,37)$ & $(6,38)(11,98)$ \\
\hline & & $15,13 \mathrm{a}$ & $7,98 \mathrm{~b}$ & $10,05 b$ & $9,19 b$ \\
\hline & & 25,29 & 29,24 & 32,82 & 19,02 \\
\hline & \multirow{3}{*}{$\begin{array}{l}\text { Espessura da parede } \\
(\mu \mathrm{m})\end{array}$} & $(0,77)(5,67)$ & $(0,89)(6,81)$ & $(0,94)(6,38)$ & $(3,62)(6,50)$ \\
\hline & & $4,48 \mathrm{ab}$ & $4,90 \mathrm{a}$ & $4,14 b$ & $4,81 \mathrm{ab}$ \\
\hline & & 17,31 & 18,14 & 22,81 & 19,86 \\
\hline
\end{tabular}

${ }^{1}$ Valores entre parênteses são mínimo e máximo, respectivamente. *Coeficiente de variação (\%). Médias seguidas pela mesma letra na horizontal não diferem estatisticamente entre si (Tukev; P>0,05).

H. dulcis, os valores mínimo e máximo variaram de 3,62 a $6,50 \mu \mathrm{m}$, respectivamente. Para as demais madeiras, os valores mínimos foram próximos de

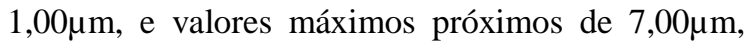
como exemplo do híbrido clonal, que indica maior amplitude de variação desta característica para estas madeiras. Classificadas como madeiras de fibras de paredes delgadas a espessas.

\section{Caracterização física}

Na tabela 2, estão apresentados os valores mínimo, máximo, médio e coeficiente de variação da densidade básica das madeiras.

O valor médio da densidade básica entre as madeiras foi estatisticamente diferente pelo teste de Tukey, com 5\% de significância. Observa-se que madeira de Hovenia dulcis apresentou a maior 
Tabela 2 - Valores da densidade básica das madeiras analisadas.

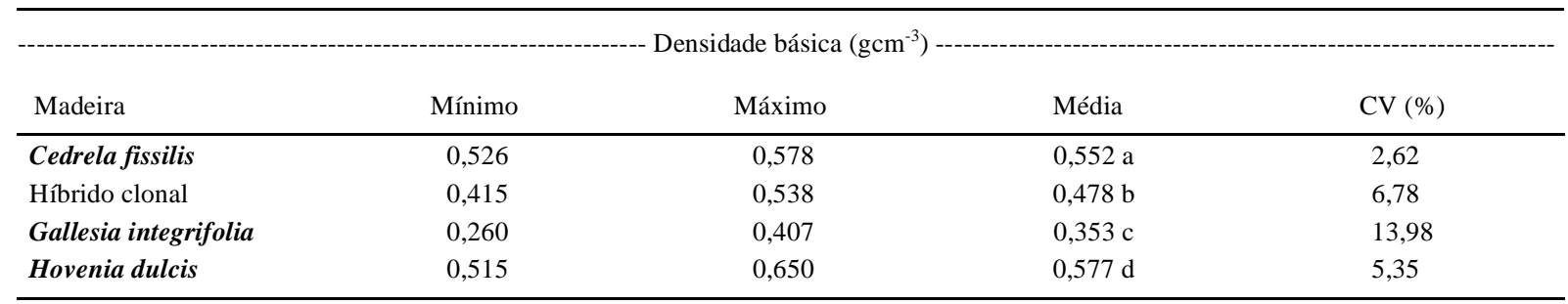

$\mathrm{CV}=$ coeficiente de variação. Médias seguidas pela mesma letra na horizontal não diferem estatisticamente entre si (Tukey, $\mathrm{P}>0,05)$.

densidade de $0,577 \mathrm{gcm}^{-3}$, seguida pelas espécies Cedrela fissilis e o Híbrido clonal com 0,552 e $0,478 \mathrm{gcm}^{-3}$, respectivamente. As três espécies podem ser classificadas como madeira de média densidade. Já madeira Gallesia integrifolia foi classificada como de baixa densidade, com uma densidade média de $0,353 \mathrm{gcm}^{-3}$.

Em função da classificação quanto à densidade básica da madeira, as espécies estudadas podem ser direcionadas de acordo com produto final e sua qualidade. Vale ressaltar também que o uso das espécies em situações que exijam maiores esforços mecânicos deve ser limitado. Verificouse que os valores determinados para a densidade básica da madeira neste estudo estão próximos de outros obtidos por diversos autores, como RIGATTO (2001), GONÇALVES (2006), VALÉRIO et al. (2008), ANDRADE (2009).

Os valores médios das contrações tangencial, radial e volumétrica e do coeficiente anisotrópico para as quatros espécies estão apresentados na tabela 3 .
De acordo com o apresentado na tabela 3, verificou-se que as contrações tangencial $(9,03 \%)$ e volumétrica $(13,75 \%)$ da madeira foram significativamente mais altas para o híbrido clonal.

A contração radial $(5,37 \%)$ da madeira de Cedrela fissilis foi a que apresentou estatisticamente o maior valor, bem como o menor valor para o coeficiente de anisotropia $(1,16)$. O baixo fator anisotrópico indica que a madeira apresenta menores propensões ao fendilhamento e empenamentos durante as alterações dimensionais, provocadas pela variação dimensional durante o processo de secagem, sendo, consequentemente, menos instáveis. De acordo com OLIVEIRA \& SILVA (2003), quanto mais próximo estiver esse valor de 1,0, maior será a estabilidade dimensional da madeira.

Para DURLO \& MARCHIORI (1992), o fator anisotrópico é o mais importante índice para se avaliar a estabilidade dimensional da madeira. Os mesmos autores apresentaram critérios de classificação da madeira quanto a esse parâmetro. Para valores entre 1,2 e 1,5, a madeira é considerada

Tabela 3 - Valores médios das contrações e coeficiente de anisotropia.

\begin{tabular}{|c|c|c|c|c|}
\hline \multirow{2}{*}{ Madeira } & 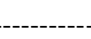 & Contração (\% & 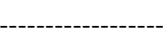 & \multirow[t]{2}{*}{ Coeficiente anisotrópico } \\
\hline & Radial & Tangencial & Volumétrica & \\
\hline \multirow{2}{*}{ Cedrela fissilis } & $5,37 \mathrm{a}$ & $6,20 \mathrm{c}$ & $11,43 \mathrm{~b}$ & $1,16 \mathrm{~B}$ \\
\hline & $2,44^{*}$ & 2,31 & 2,13 & 3,43 \\
\hline \multirow{2}{*}{ Híbrido clonal } & $5,02 \mathrm{~b}$ & $9,03 \mathrm{a}$ & $13,75 \mathrm{a}$ & $1,81 \mathrm{~A}$ \\
\hline & 10,66 & 5,17 & 6,31 & 8,65 \\
\hline \multirow{2}{*}{ Gallesia integrifolia } & $3,56 \mathrm{c}$ & $6,54 \mathrm{c}$ & $10,13 \mathrm{c}$ & $1,84 \mathrm{~A}$ \\
\hline & 7,40 & 11,99 & 8,91 & 10,98 \\
\hline \multirow{2}{*}{ Hovenia dulcis } & $4,36 \mathrm{~d}$ & $7,68 \mathrm{~b}$ & $11,76 \mathrm{~b}$ & $1,77 \mathrm{~A}$ \\
\hline & 6,44 & 2,47 & 2,77 & 6,71 \\
\hline
\end{tabular}

* Coeficiente de variação (\%). Médias seguidas pela mesma letra na horizontal, não diferem estatisticamente entre si (Tukey, P>0,05). 
excelente, como a madeira de cedro, sucupira, mogno, balsa; valores entre 1,5 e 2,0, a madeira é considerada normal, como o ipê, pinus, araucária, peroba-rosa e teca; e, acima de 2,0, a madeira é considerada ruim, exemplo da imbúia, álamo e jatobá. Portanto, em função desta classificação, a madeira da espécie Cedrela fissilis é classificada como excelente, e as madeiras das espécies do híbrido clonal, Gallesia integrifolia e Hovenia dulcis são classificadas como normais. Madeiras que apresentam elevada estabilidade dimensional podem ser utilizadas normalmente na fabricação de piso, esquadrias, portas e móveis.

Segundo CARVALHO (1994), a espécie Hovenia dulcis apresenta potencial de utilização diverso, entre eles a construção civil, marcenaria, vigas, caibros, tábuas, assoalhos, moirões, utilizada também na fabricação de móveis e laminados, além do seu aproveitamento na indústria de celulose e papel.

A madeira Cedrela fissilis pode ser largamente empregada em compensados, contraplacados, esculturas e obras de talha, modelos e molduras, esquadrias, móveis em geral, marcenaria, na construção civil, naval e aeronáutica, na confecção de pequenas caixas, lápis e instrumentos musicais (LORENZI, 1996).

A espécie Gallesia integrifolia possui madeira de baixa densidade e alta instabilidade dimensional, suas aplicações são restritas e normalmente utilizadas na obtenção de tábuas e sarrafos, que podem ser utilizados em cimbramento, construções temporárias, caixotaria, embalagens leves e miolo de painéis (MAINIERI \& CHIMELO, 1989).

\section{CONCLUSÃO}

Com base nos resultados apresentados e discutidos com relação às espécies estudadas, verificouse que as madeiras foram classificadas como leve e de média densidade básica. A madeira de Cedrela fissilis demonstrou maior estabilidade dimensional com o comportamento classificado em excelente.

As madeiras de Hovenia dulcis e do Híbrido clonal foram indicados para uso na construção civil. A madeira de Gallesia integrifolia apresentou baixa densidade básica e estabilidade dimensional.

\section{AGRADECIMENTOS}

À Fundação de Apoio à Ciência e Tecnologia do Espírito Santo (FAPES) pela concessão de auxílio financeiro na forma de uma bolsa de estudo e ao Laboratório de Ciência da
Madeira (LCM) do Núcleo de Estudos e de Difusão de Tecnologia em Florestas, Recursos Hídricos e Agricultura Sustentável (NEDTEC) do Centro de Ciência Agrárias (CCA) da Universidade Federal do Espírito Santo (UFES).

\section{REFERÊNCIAS}

ALBUQUERQUE, C.E.C. et al., Adesão e adesivos. In: IWAKIRI, S. Painéis de madeira reconstituída. Curitiba: FUPEF. 247p. 2005.

ANDRADE, I.M; et al. Efeito da procedência na variação radial do comprimento de fibras e densidade básica da madeira de Gallesia integrifolia (Spreng.) Harms. IF Série Registro, São Paulo, v.40, p.39-43, 2009.

BREMER, C.F. Histórico das construções de madeira. In: RODRIGUES, B.P.; FIEDLER, N.C.; BRAZ, R.L. Tópicos em ciências florestais. n.1. Alegre: CCAUFES. p.11-21, 2009.

CARVALHO, P.E.R. Ecologia, silvicultura e usos da uva-do-japão (Hovenia dulcis Thunberg). Colombo: EMBRAPA-CNPF, 1994. 24p. (Circular técnica, 23). Disponível em: <http://www.infoteca. cnptia.embrapa.br/handle/doc/290745>. Acesso em: 10 ago. 2012.

COMMISSION PANAMERICANA DE NORMAS TÉCNICAS - COPANT. Descripcion de características generales, macroscópicas de las maderas angiospermas dicotiledoneas. v.30. p.1-19. 1974.

COSTA, A. Coletâneas de anatomia da madeira. Anatomia da madeira. 42p. 2001. Disponível em: <http://www.joinville.udesc. br/sbs/professores/arlindo/materiais/APOSTILANATOMIA1. pdf $>$. Acesso em: 10 ago 2012.

CHIMELO, J. Anatomia e propriedades gerais da madeira. In: OLIVEIRA, J.T.S.; FIEDLER, N.C.; NOGUEIRA, M. Tecnologias aplicadas ao setor madeireiro. Jerônimo Monteiro: Suprema Gráfica e Editora. p.107-124. 2007

CRUZ, C.R. et al. Variações dentro das árvores e entre clones das propriedades físicas e mecânicas da madeira de híbridos de Eucalyptus. Scientia Forestalis, Piracicaba, n.64, p.33-37, 2003. Disponível em: <http://www.ipef.br/publicacoes/scientia/nr64/ cap03.pdf>. Acesso em: 10 ago. 2012.

DURLO, M.A; MARCHIORI, J.N.C. Tecnologia da madeira: retratibilidade. Santa Maria: CEPEF/FATEC, 1992. 33p. (Série Técnica, 10).

FREDERICO, P.G.U. Efeito da região e da madeira de eucalipto nas propriedades do carvão vegetal. 2009. 85f. Dissertação (Mestrado em Ciência Florestal) - Universidade Federal de Viçosa, Viçosa, MG.

GONÇALVES, F. Avaliação da qualidade da madeira de híbrido clonal de Eucalyptus urophylla $\mathbf{x}$ Eucalyptus grandis para produtos sólidos. 2006.169f. Dissertação (Mestrado em Produção vegetal) - Universidade Federal do Espírito Santo, Alegre, ES.

IAWA COMMITTEE. List of microscopic features for hardwood identification, with an appendix on non-anatomical information. IAWA Bulletin, Leiden, v.10, n.3, p. 219-332. 1989.

LATORRACA, J.V.; ALBUQUERQUE, C.E.C. Efeito do rápido crescimento sobre as propriedades da madeira. 
Revista Floresta e Ambiente, Seropédica, v.7, n.1, p.279291, 2000. Disponível em: <http://www.floram.org/files/ v7n\%C3\%BAnico/v7nunicoa29.pdf>. Acesso em: 10 ago. 2012.

LOBÃO, M.S. et al. Caracterização das propriedades físico-mecânicas da madeira de eucalipto com diferentes densidades. Revista Árvore, Viçosa, v.28, n.6, p.889-894, 2004. Disponível em: <http://dx.doi.org/10.1590/S010067622004000600014>. Acesso em: 15 ago. 2014. doi: 10.1590/ S0100-67622004000600014.

LORENZI, H. Árvores brasileiras: manual de identificação e cultivo de plantas arbóreas nativas do Brasil. São Paulo: Plantarum, 1996. V.1, 135p.

MAINIERI, C.; CHIMELO, J.P. Fichas de características das madeiras brasileiras. São Paulo: IPT, 1989. 418p.

RAMALHO, R.S. O uso de macerado no estudo anatômico de madeiras. Viçosa, MG: UFV, 4p, 1987.
RIGATTO, P.A. et al. Características físicas, químicas e anatômicas da madeira de Hovenia dulcis. Colombo: EMBRAPA, 2001. 4p. (Comunicado técnico, 66). Disponível em: <http://www.infoteca.cnptia.embrapa.br/handle/doc/304710>. Acesso em: 14 ago. 2012.

SILVA, J.C; OLIVEIRA, J.T.S. Avaliação das propriedades higroscópicas da madeira de Eucalyptus saligna, em diferentes condições de umidade relativa do ar. Revista Árvore, Viçosa, v.27, n.2, p.233-239, 2003. Disponível em: <http://dx.doi.org/10.1590/ S0100-67622003000200012>. Acesso em: 14 ago. 2012. doi: 10.1590/S0100-67622003000200012.

TOMAZELLO FILHO, M. Formação e anatomia da madeira. Piracicaba: ESALQ/USP, 2002. 76p. (Apostila)

VALÉRIO, A.F. et al. Determinação da densidade básica da madeira de cedro (Cedrela fissilisVell.) ao longo do fuste. Pesquisa aplicada e agrotecnologia, v.1, n.1. p.23-28, 2008. Disponível em: <http://revistas.unicentro.br/index.php/repaa/article/view/5>. Acesso em: 15 ago. 2012. doi: 10.5777/paet.v1i1.5.

Ciência Rural, v.44, n.12, dez, 2014. 